\title{
Segmental arterial mediolysis, reparative phase: An analysis and case report showing conversion to fibromuscular dysplasia with renal infarction
}

\author{
Richard E. Slavin ${ }^{\text {* }}$, Julian del Cerro Gonzalez ${ }^{2}$, Jose Manuel Machin ${ }^{3}$, Angel Robles ${ }^{4}$, \\ Rita Maria Regojo ${ }^{5}$, Marie Luisa Diez ${ }^{6}$ \\ ${ }^{1}$ Department of Pathology, Legacy Emanuel Hospital, Portland, Oregon, USA \\ ${ }^{2}$ Interventional Radiologist, University Hospital, Guadalajara, Spain \\ ${ }^{3}$ Department of Internal Medicine, University Hospital, Guadalajara, Spain \\ ${ }^{4}$ Department of Internal Medicine, La Paz University Hospital, Madrid, Spain \\ ${ }^{5}$ Department of Pathology, University Hospital, Guadalajara, Spain \\ ${ }^{6}$ Family Practice Physician, Guadalajara Health Center, Cervantes Branch, Guadalajara, Spain \\ Email: *rslavin@lhs.org, reslavin@comcast.net
}

Received 30 November 2013; revised 31 December 2013; accepted 10 January 2014

Copyright (C) 2014 Richard E. Slavin et al. This is an open access article distributed under the Creative Commons Attribution License, which permits unrestricted use, distribution, and reproduction in any medium, provided the original work is properly cited. In accordance of the Creative Commons Attribution License all Copyrights (c) 2014 are reserved for SCIRP and the owner of the intellectual property Richard E. Slavin et al. All Copyright (C) 2014 are guarded by law and by SCIRP as a guardian.

\section{ABSTRACT}

Segmental arterial mediolysis (SAM), an uncommon arteriopathy putatively caused by norepinephrine released by alpha-1 adrenergic agonists or some Beta-2 agonists capable of releasing norepinephrine from the peripheral sympathetic nervous system potentially can present ischemic and organ injury symptoms caused by sequelae created in its reparative phase in lieu of catastrophic hemorrhages announced in its injurious phase. The case documents this presentation-the patient presenting renal infarcts and ischemic lesions causing abdominal angina, hypertension and a nephrectomy event developing 10 years after prolonged ritodrine treatment for premature labor. This agent may have directly caused SAM or sensitized the patient to agonists causing SAM encountered at a latter date. A variety of lesions derived from injurious phase arterial injuries characterize reparative phase SAM. All were encountered in a hilar branch of the resected renal artery. These included side-by-side sequela aneurysms grossly forming a large fusiform aneurysm, granulation tissue filling adventitial medial tear spaces in which a dissecting hematomas developed, medial muscle loss centered to the outer media repaired with fibrous tissue, arterial stenosis created by reparative intimal plaques, and arterial thrombo-embolism. These lesions were mirrored in

${ }^{*}$ Corresponding author. accompanying radiologic studies. The accompanying renal vein exhibited changes consistent with repair of the spastic venous angiopathy that often accompanies abdominal SAM. This angiopathy, putatively induced by Endothelin-1, suggested that this agent played a role in the genesis of the arterial lesions. Angiographic resolution of non-treated sequelae occurred in 5 months either spontaneously or due to treatment with bosentem. Conclusions: The histologic and angiographic changes demonstrate that the clinical onset of reparative SAM may be significantly delayed to produce ischemic lesions, renal infarction and in this case report, medial fibromuscular dysplasia in the hilar branch of the renal artery.

\section{KEYWORDS}

Segmental Arterial Mediolysis; Fibromuscular Dysplasia; Ritodrine; Renal Infarcts; Dissecting Hematoma; Intestinal Angina; Endothelin-1; Bosentem

\section{INTRODUCTION}

Catastrophic hemorrhage occurring in the abdominal cavity and/or at the brain base is the most striking and recognized symptom announcing segmental arterial mediolysis (SAM), an uncommon potentially lethal arteriopathy believed to be principally caused by an iatrogenic or accidental exposure to alpha-1 adrenergic receptor 
agonists or to B-2 agonists able to release norepinephrine from the peripheral nervous system [1,2]. On the basis of histological and radiologic studies that reported nonsymptomatic lesions in the injurious phase of SAM, it was postulated that SAM might also present in another fashion-as an ischemic disorder alone or associated with organ injury created by sequelae stemming from these non-symptomatic injurious lesions [3]. This article will describe a case of SAM presenting with the latter characteristics-renal infarcts and ischemic manifestations created by sequelae putatively developing years after treatment with or sensitization by ritodrine a Beta-2 tocolytic drug believed capable of releasing norepinephrine from the peripheral sympathetic nervous system.

\section{CASE PRESENTATION}

The patient, a female physician, pregnant at age of 38, developed premature labor in her 19th week of pregnancy. She was treated for this with the tocolytic agent ritodrine for the following 17 weeks. The ritodrine was administered at a dosage of $10 \mathrm{mg}$ every 4 hours except for a 20-day period of intravenous dosage starting in the 26 weeks of pregnancy. The total IV dosage is unknown. During treatment with ritodrine she exhibited bouts of tachycardia, flushing and tremor. Her delivery was uncomplicated and the delivered infant boy did not exhibit any detectable abnormalities.
Ten years after delivery, the patient suddenly developed severe pain in the left lumbar fossa radiating to the abdomen lasting for about 36 hours. This was initially attributed to renal colic. Six days later the pain recurred and was now localized to the epigastric and mesogastric regions. This prompted hospital admission. The pain improved with bowel rest, analgesics and treatment with omeprazole. Laboratory blood tests revealed a moderate elevation in Lactate dehydrogenase and hematuria. Radiological studies were performed 11 and 21 days after the onset of symptoms. These showed a left renal infarct and the vascular abnormalities illustrated and described in Figures 1 to 5 . On the bases of the laboratory findings and the renal radiologic studies a diagnosis a left renal infarct was established and a diagnosis of SAM was made supported by the intestinal angiographic findings.

The day following the first radiologic study, the patient was readmitted to hospital because of the recurrence of severe abdominal pain. This was attributed to recurring renal infarcts from emboli from the lobar branch of the left renal artery and was treated with catheterizing and an attempted placement of stents in the left upper renal artery together with fibrinolysis. Unfortunately successful endoluminal treatment by the vascular surgeons could not be accomplished and the placement of the stent in a subintimal position caused a dissection. The worsening of symptoms and the elevation of her blood pressure provoked a left nephrectomy that was performed 39

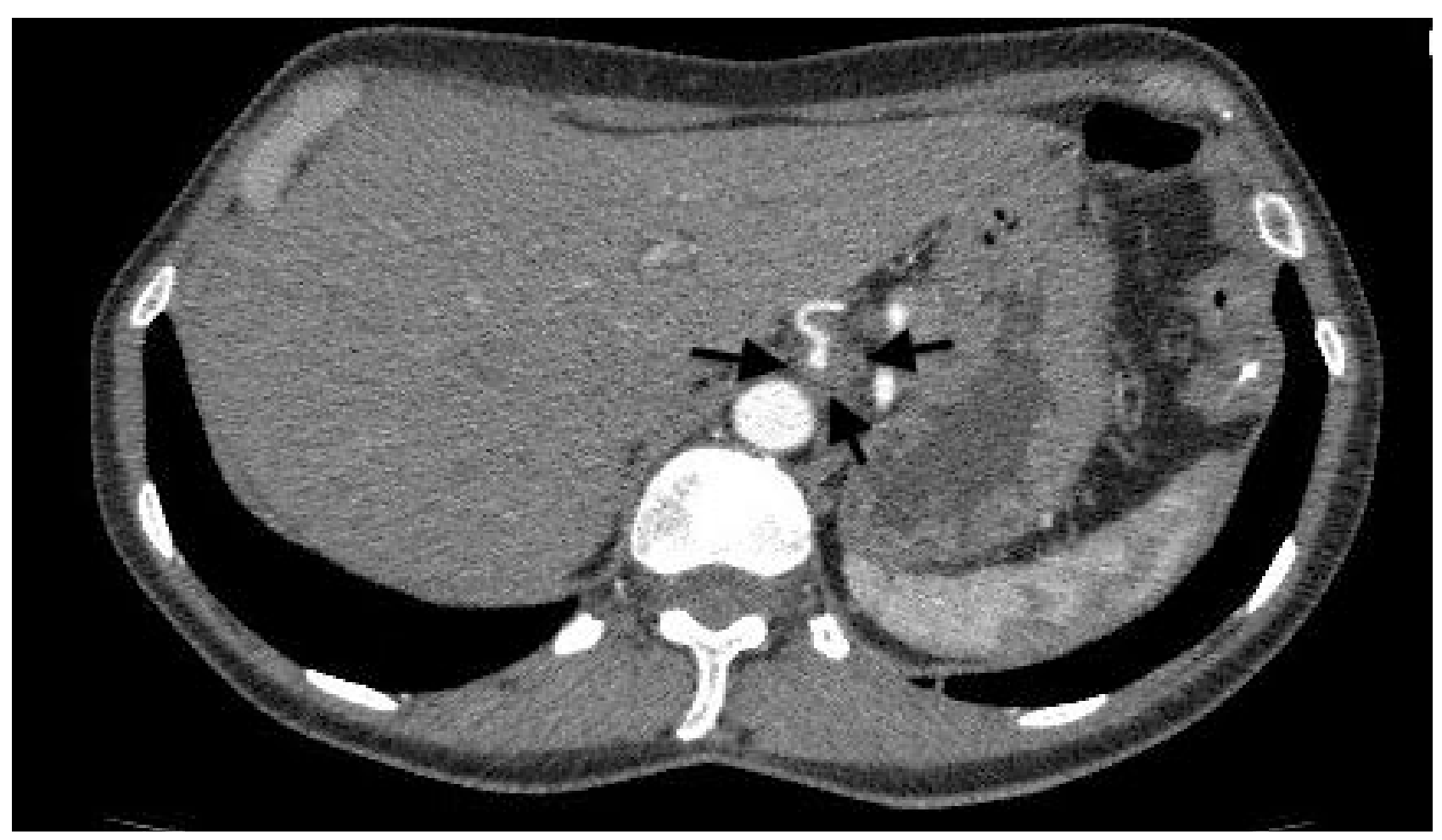

Figure 1. Axial enhanced CT Arrows outline a cuff image around the celiac trunk. 


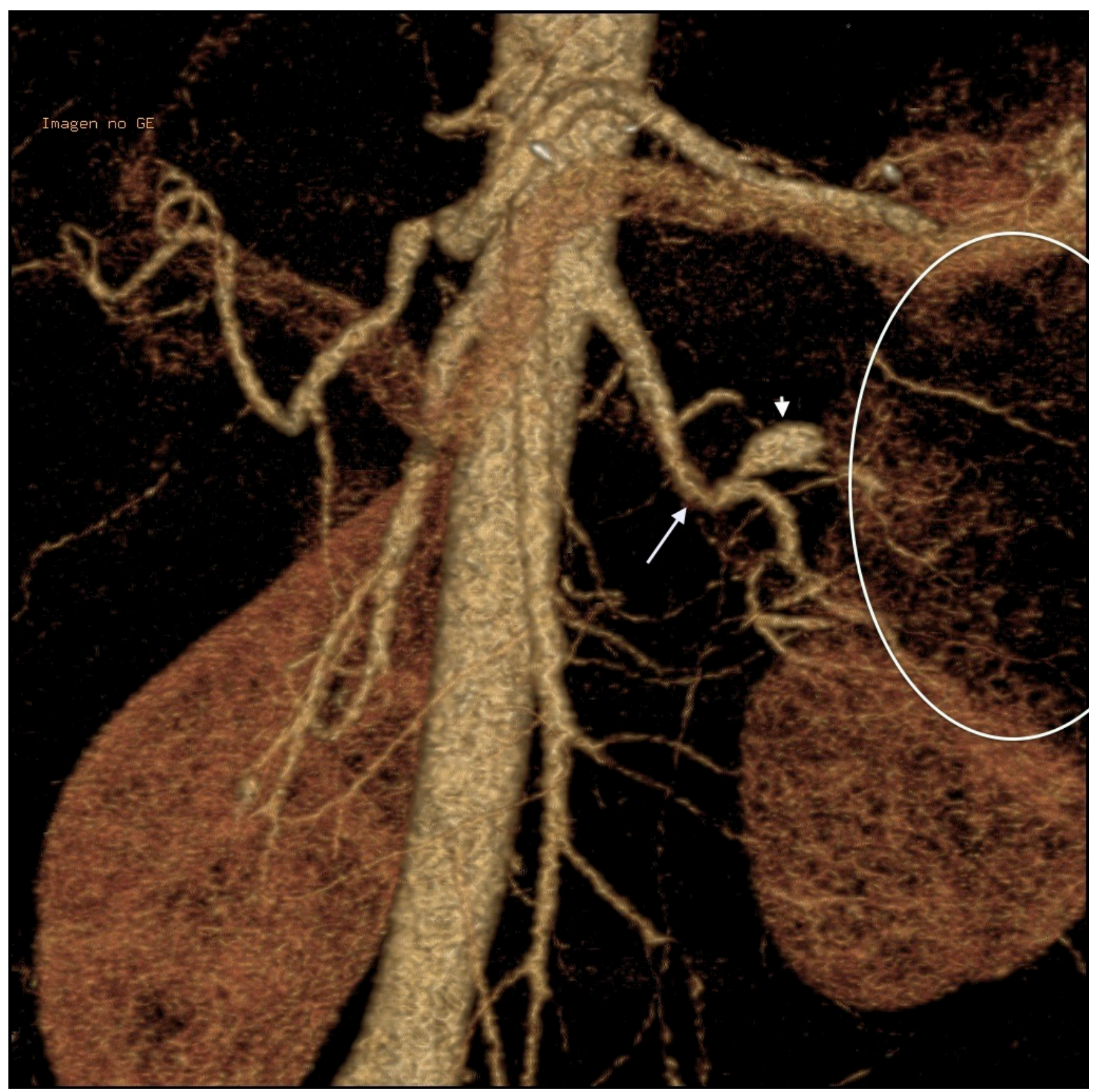

Figure 2. 3D CT reconstruction of abdominal aorta showing mild stenosis before bifurcation of the left renal artery (oblique arrow), an aneurysm of a superior polar branch (arrow head) and a left kidney superior pole infarct (ellipse).

days after the onset of symptoms. Three weeks post nephrectomy abdominal pain recurred caused by a right renal infarct. This was small without any impact on renal function or blood pressure and for these reasons intervention was not attempted. She was treated with oral anticoagulants. The patient continued to have episodes of abdominal pain treated with analgesics, suspected as representing abdominal angina. A year later the patient was placed on a Bosentan regimen and she states that her symptoms have since improved and her blood pressure is no longer elevated. Cardiac studies performed on the patient revealed no abnormalities. Five months after her bosentem was initiated repeat angiographic studies showed resolution of remaining arterial sequelae (Figures 6 and 7) and the return of well being in the patient except for the occasional occurrence of minor episodes of abdominal pain.

\section{Morphology}

Sections of the kidney show a focal organizing infarct. An adjacent interlobular artery contains an organizing 


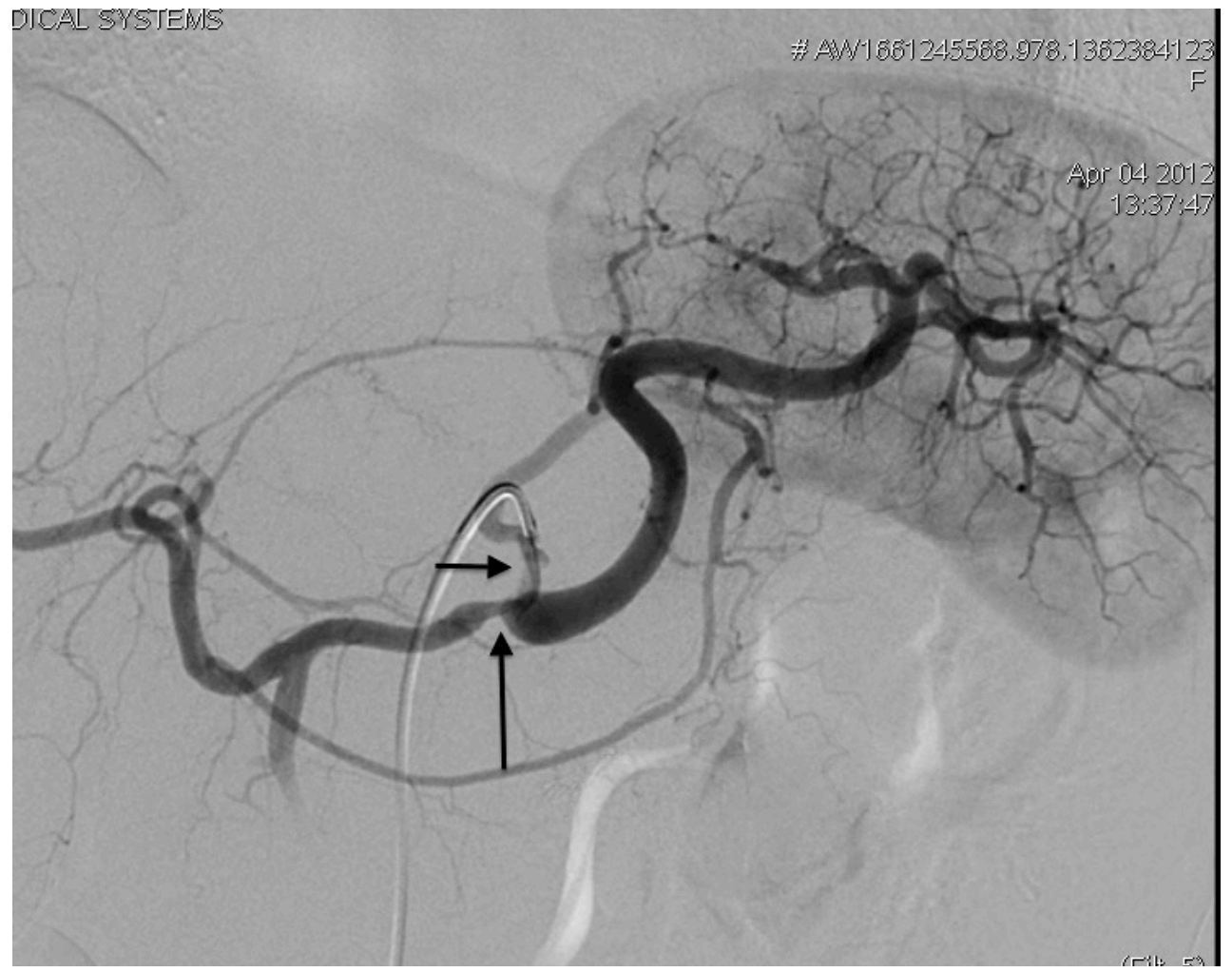

Figure 3. Celiac trunk angiography shows stenosis and a small pseudoaneurysm of the celiac trunk (horizontal arrow) and stenosis in the origin of the common hepatic artery (vertical arrow).

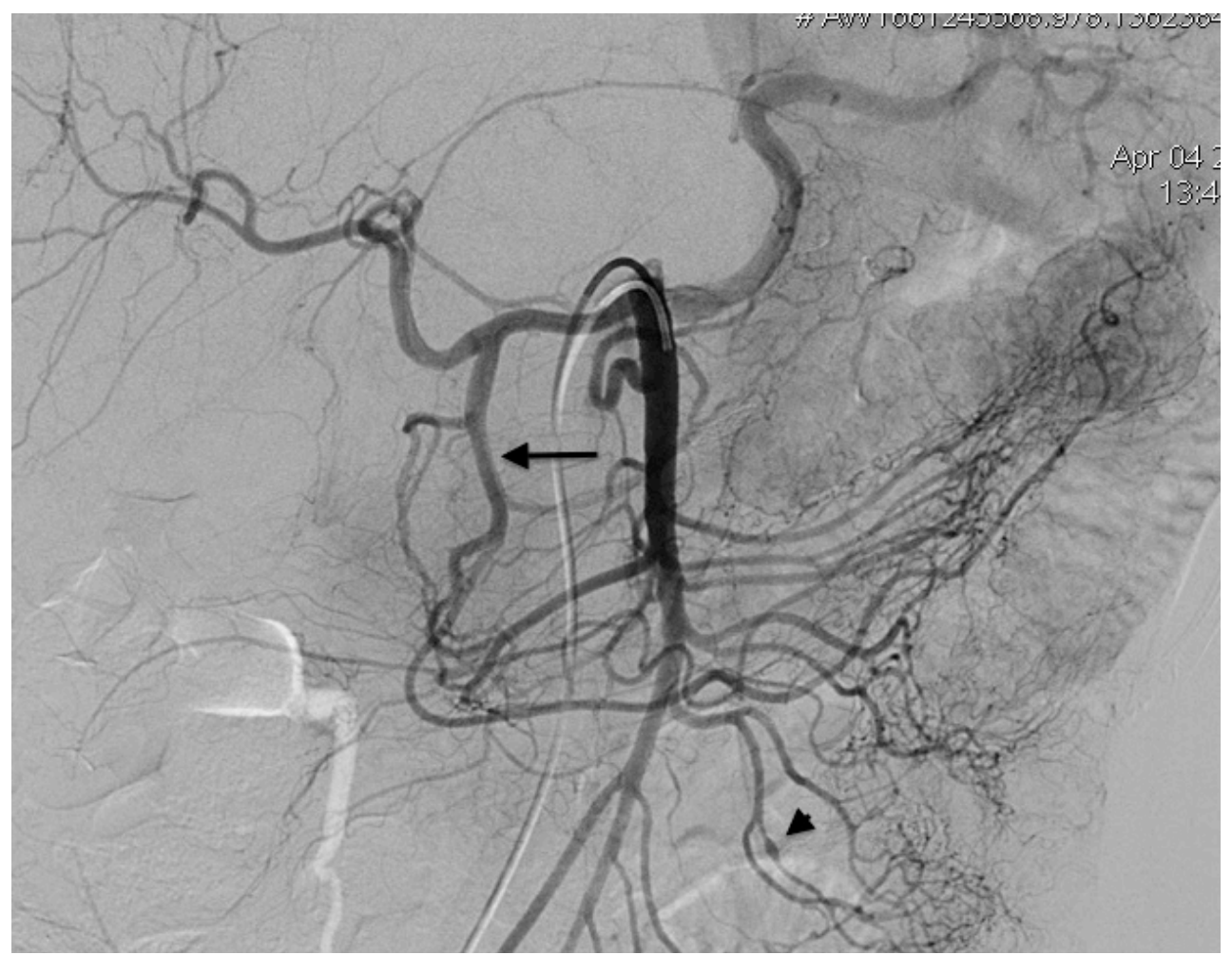

Figure 4. Superior mesenteric angiography shows a small aneurysm (arrow head) in a distal branch of an ileal artery and a collateral circulation through the gastroduodenal artery extending toward the hepatic and splenic arteries (horizontal arrow). 


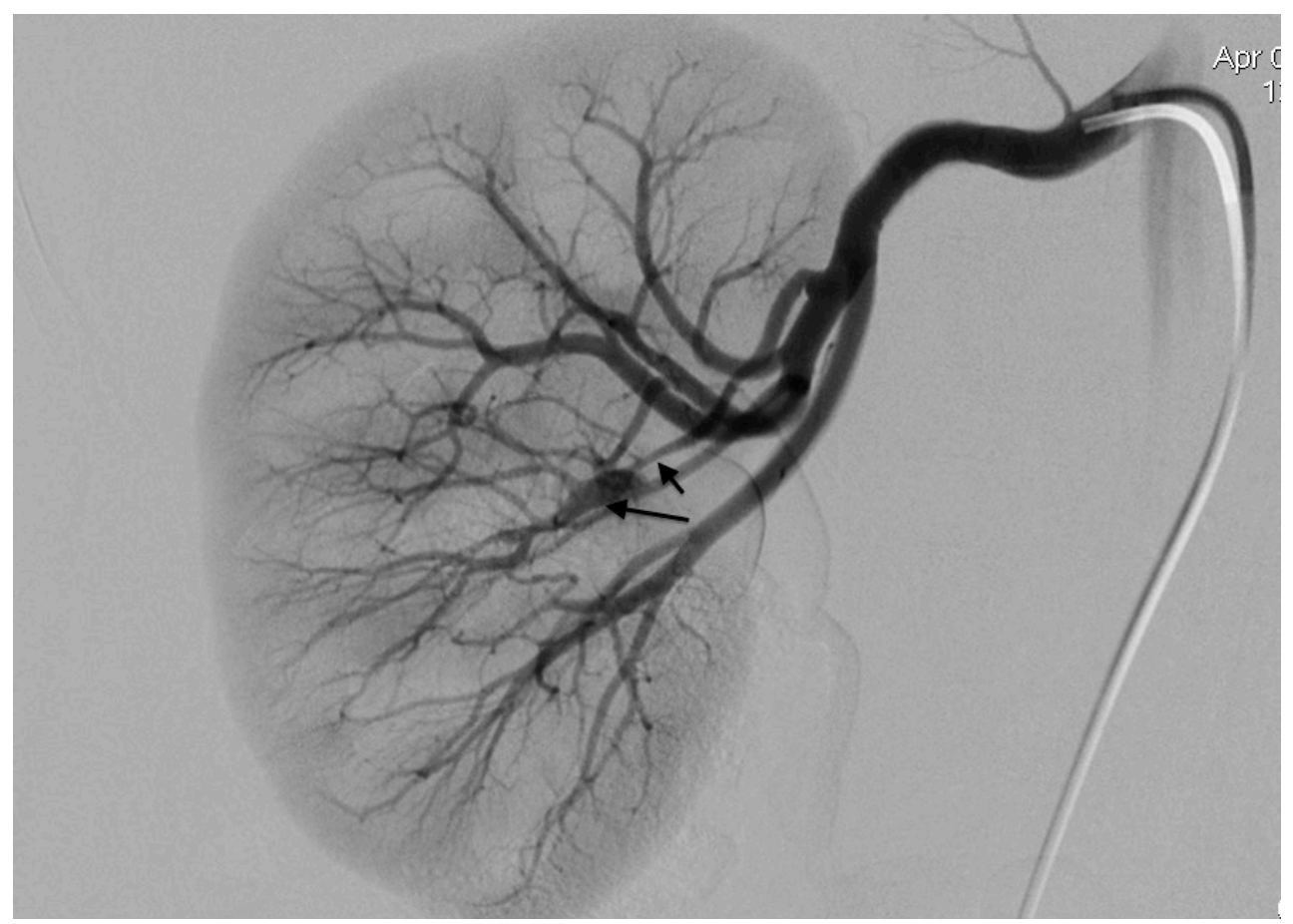

Figure 5. Right renal angiography showing stenosis (small arrow) and an aneurysm (large arrow) of a medial branch near the renal hilum.

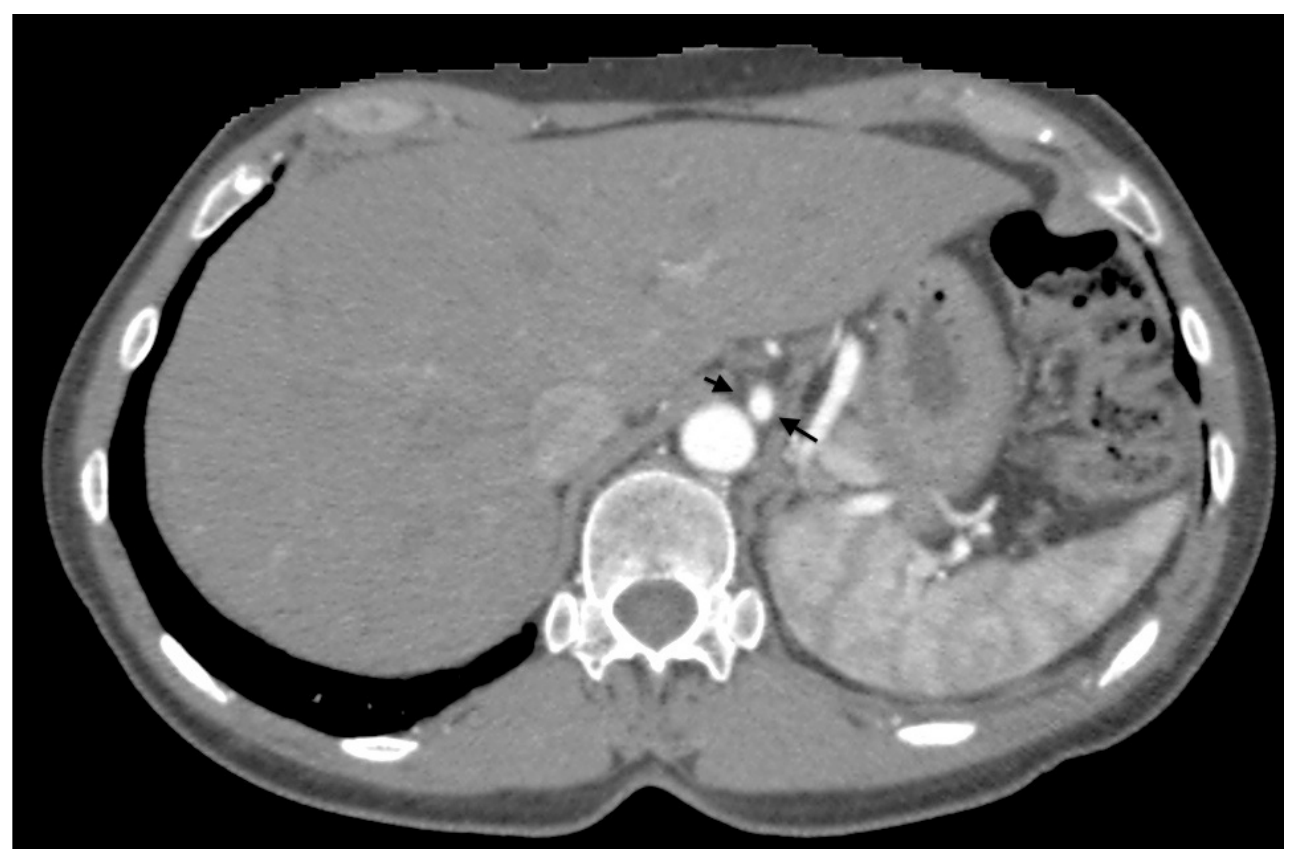

Figure 6. Axial CT of celiac trunk showing the disappearance of the cuff image around the celiac trunk (arrows) seen in Figure 1.

thrombo-embolus and a recent embolus is evident in a hilar artery. The arterioles do not show hypertensive changes and no other renal abnormalities are evident.

The hilar branch of the renal artery exhibits two sideby side aneurysms whose walls are stoutly reinforced by mature granulation tissue that in one has focally ex- tended over the contiguous artery wall to form an intimal plaque (Figure 8). The intact arterial wall immediately adjacent to both aneurysms exhibits tearing separations of the outer media from the adventitia repaired by wedges of mature granulation tissue (Figures 8 and 9). The outer medial smooth muscle abutting the granulation tissue 


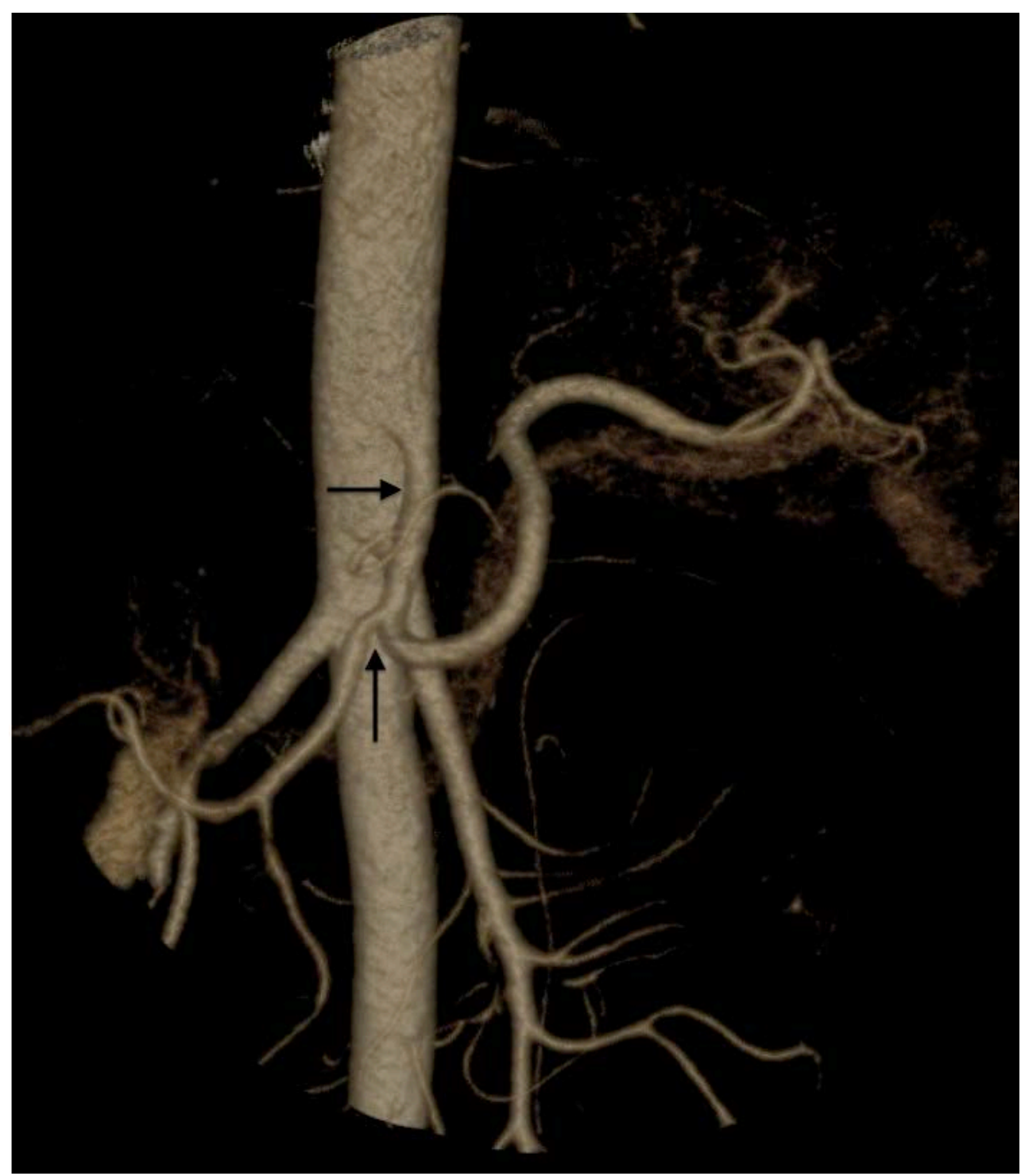

Figure 7. 3D CT. Horizontal arrow showing disappearance of the stenosis and pseudoaneurysm of the celiac trunk and vertical arrow showing minimal stenosis of the hepatic artery.

shows patchy linear loss with fibrous tissue replacement that focally extends into the mid-media (Figure 9).

Focal areas of acute hemorrhage are evident in granulation tissue developed between the outer media and adventitia (Figure 10) that extends into a dissecting hematoma formed between the outer medial wall and the adventitia of a branching hilar artery (Figure 11). The medial wall bordering the pseudolumen shows patchy fibroblastic replacement of lost smooth muscle cells with collagen formation. A thick intimal plaque composed of mature granulation tissue overrides areas of medial repair to significantly narrow the true arterial lumen.

The adjoining renal vein displays areas of luxuriant fibrosis of the outer media that separates and replaces smooth muscle bundles widening the vein (Figure 12).

A thin elastic fiber containing plaque incompletely overlies the intima.

\section{DISCUSSION}

SAM is a biphasic clinical disease. Its most recognized presentation is striking and potentially catastrophic abdominal hemorrhages. This principally develops without sexual preference in late middle-aged or elderly patients and is caused by ruptured gap-aneurysms [1]. This lesion, created in the initial injurious phase of SAM, is derived from apoptotic lysis of the arterial medial muscle with concomitant loss of the arterial intima to form arterial gaps. Enlarged gaps caused by areas of extensive mediolysis or the detachment of adjacent medial islands then become aneurysms. There selective development in older patients is putatively attributable to aging degenerative 


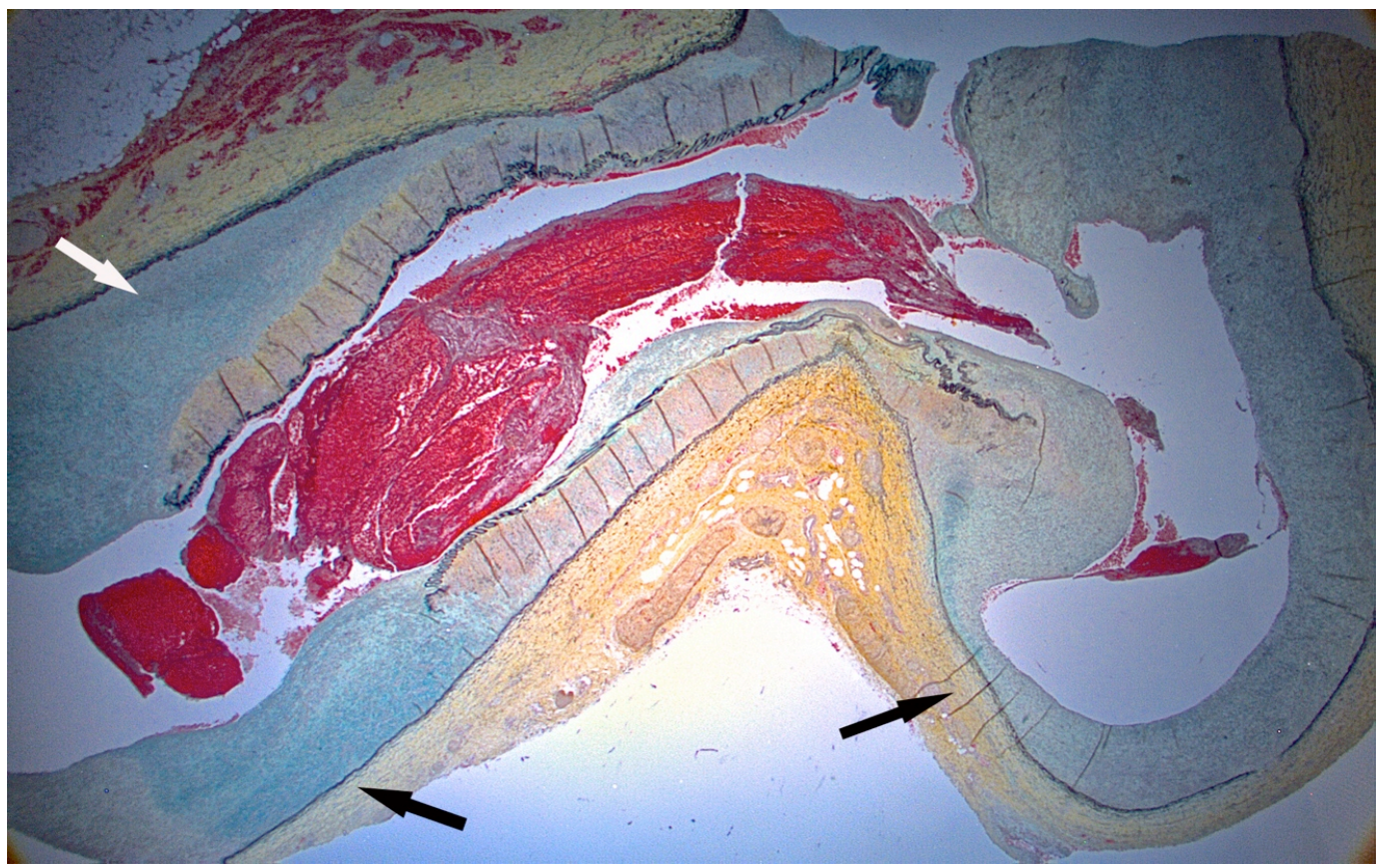

Figure 8. Reparative phase of segmental arterial mediolysis. Renal artery shows two side-by-side aneurysms (black arrows) both buttressed by a thick layer of mature granulation tissue that extensively extends into and fills the adventitial-medial tear of the upper wall of the left aneurysm (white arrow) and bedecks the intima of the lower arterial wall. Movat stain $\times 10$.

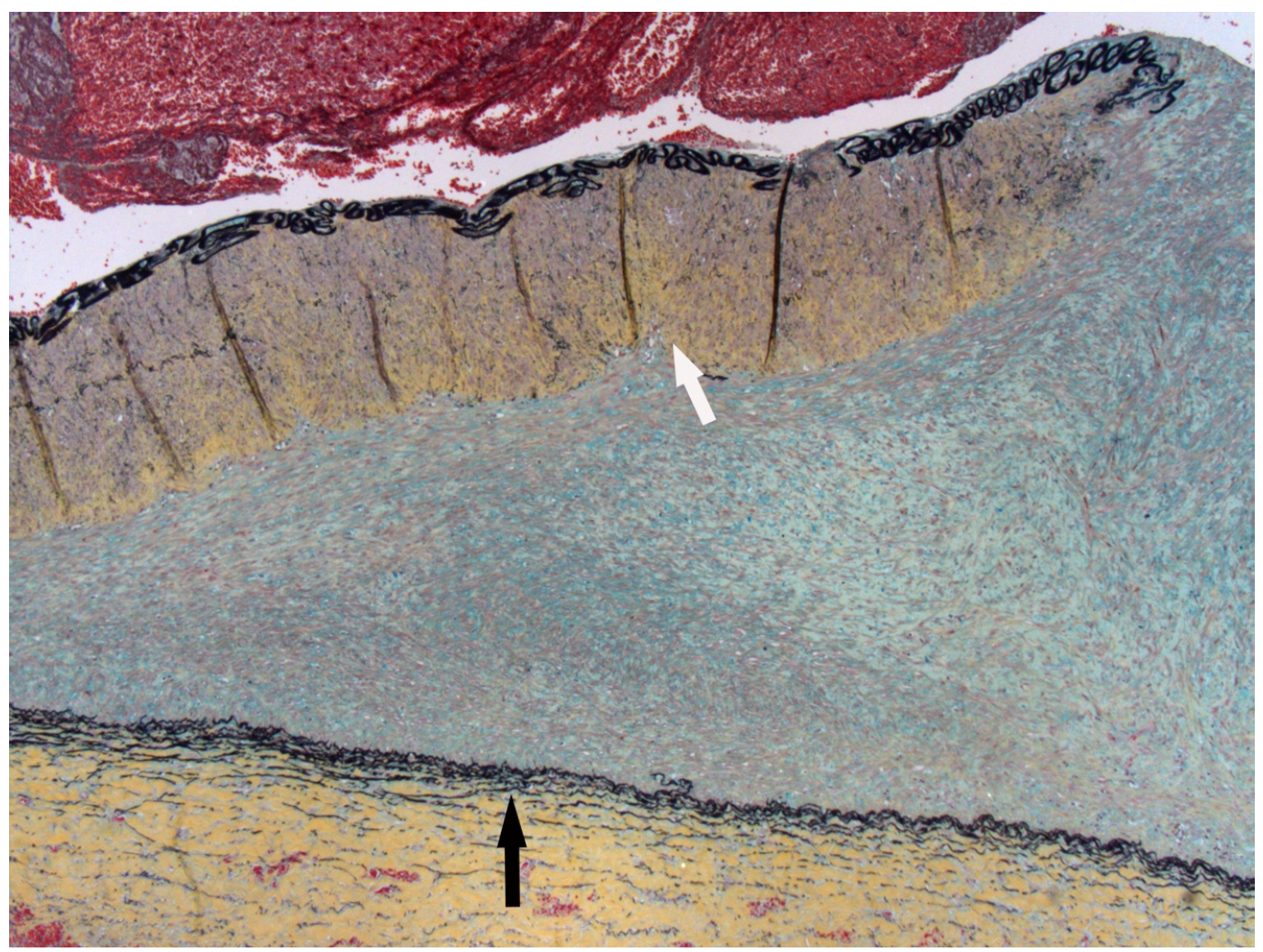

Figure 9. Reparative phase of segmental arterial mediolysis. Higher magnification of the tear separation of the media from the adventitia filled with mature granulation tissue evident in Figure 5. Detached arterial wall shows fibrosis (yellow color) of outer media focally extending into mid-media (white arrows). Lower segment of tear outlined by the external elastica (black arrow). Movat stain $\times 50$. 


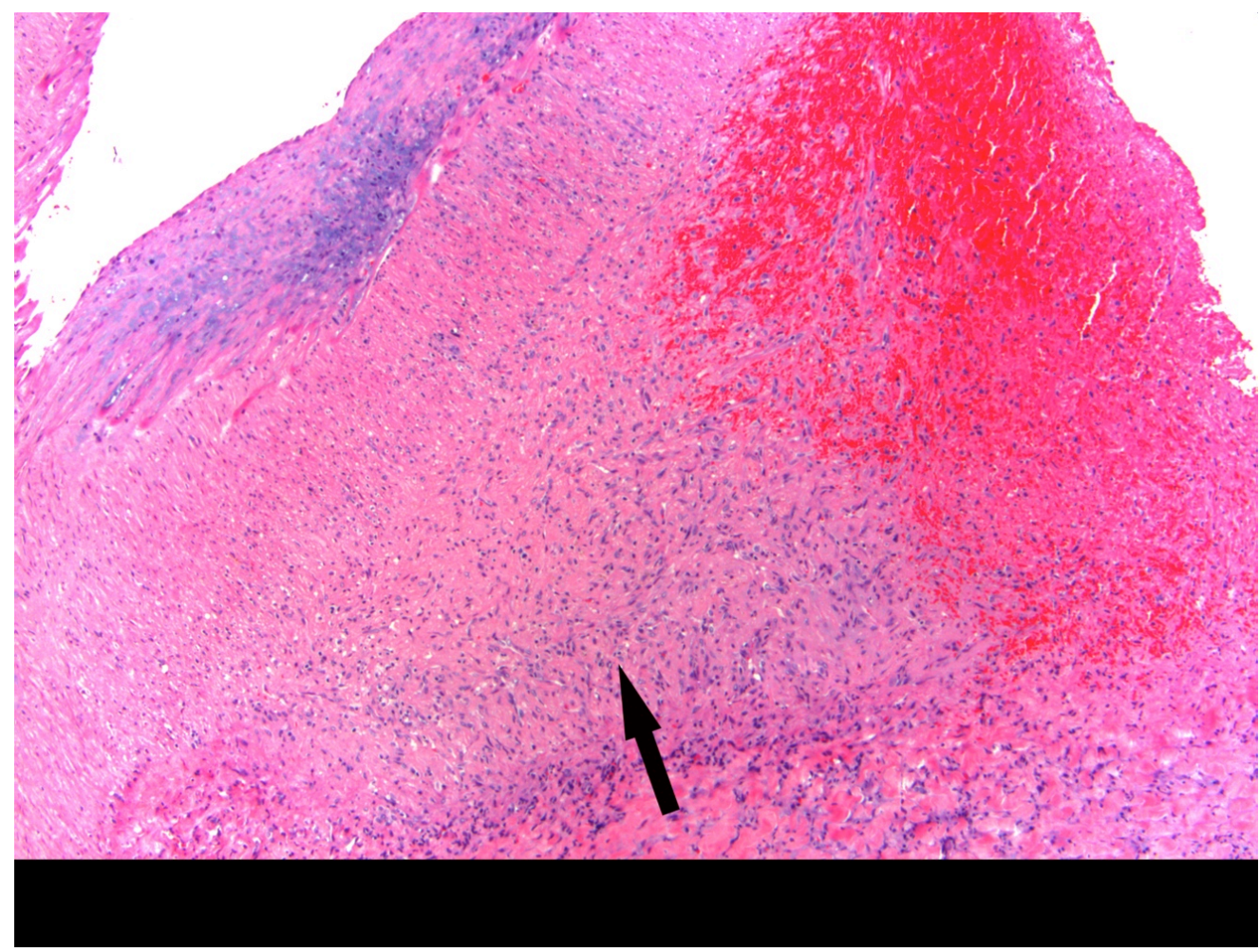

Figure 10. Granulation tissue (arrow) formed in tear space between arterial wall and adventitia showing recent hemorrhage. H\&E stain $\times 100$.

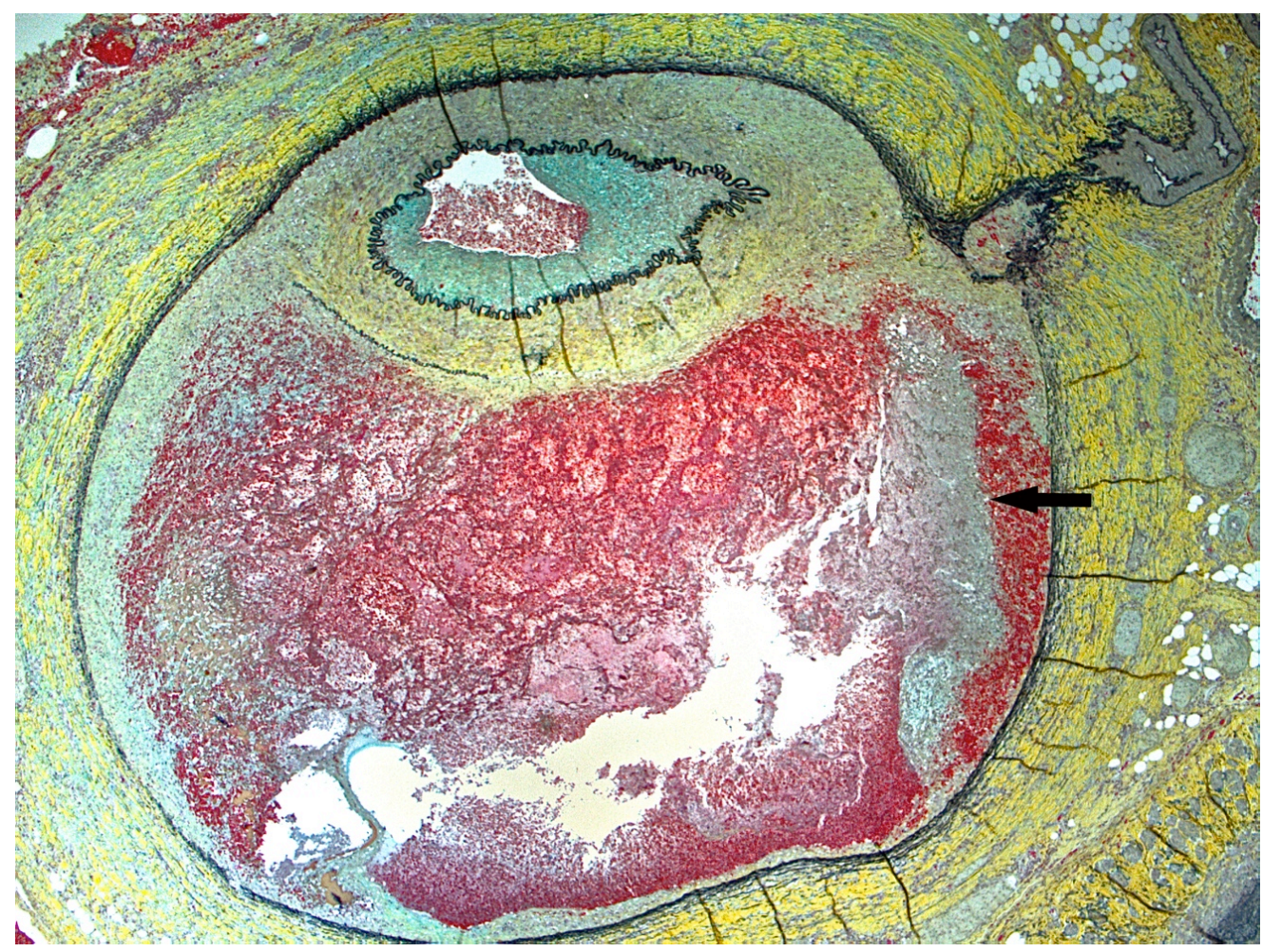

Figure 11. Reparative phase of segmental arterial mediolysis showing a dissecting hematoma developed between the outer media and adventitia. Arrow indicates an area of bleeding in recent granulation tissue in the pseudo lumen. Outer medial wall facing the dissection is fibrotic (yellow). A plaque of granulation tissue narrows the true lumen. Movat stain $\times 15$. 


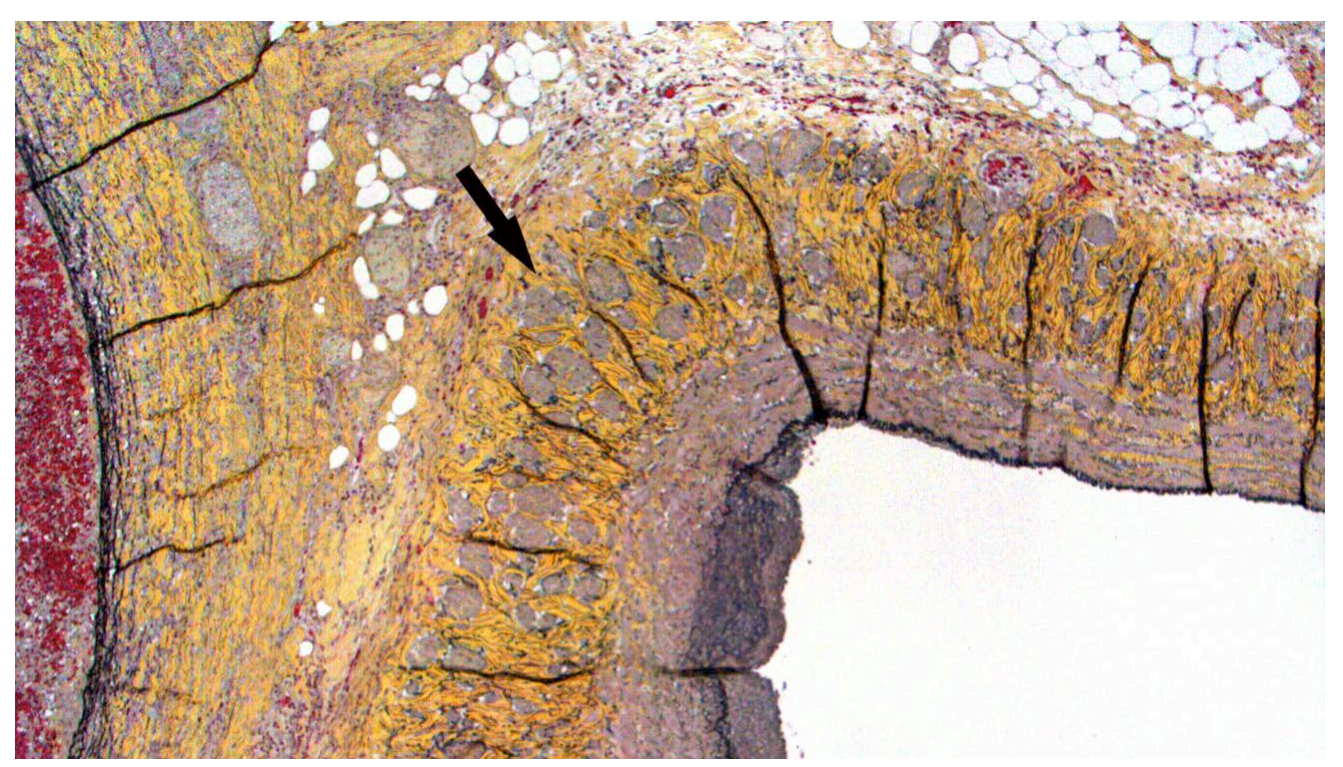

Figure 12. Venous angiopathy of segmental arterial mediolysis. Arrow points to renal vein adjacent to dissecting hematoma that shows fibrosis (yellow tissue) of the outer media developing between and separating isolated smooth muscle bundles (brown tissue) causing thickening of the venous wall. Small plaques containing elastic fibers bedeck intima. Movat stain $\times 50$.

changes that weaken the muscle-stromal connections thereby facilitating medial island separation, a heightened sensitivity to sympathomimetic agonists and the usage of these agents to combat cardiogenic and septic shock conditions not infrequent in the elderly. However, other arterial lesions occur alone or concurrently with gap aneurysms. These are areas of mediolysis of lesser intensity with preserved intima and foci of adventitial-medial separation putatively induced by violent medial muscle contraction and/or an apoptotic induced tear at this site. These two lesser injuries and small gaps may be initially asymptomatic due to the rapidly forming reparative granulation tissue that evolves as a response to the injurious lesions, and initiates the second reparative phase of SAM $[1,3,4]$. This vigorous reparative response and the absence of gap-aneurysms accounts for the paucity of clinical reports of injurious phase abdominal SAM in young adults. These asymptomatic lesions may resolve, persist or develop into sequelae that cause ischemic lesions and organ injury [3].

Sequelae are created in both the adventitial-medial junction tear site and in the media. In the former location the small vessel component of the reparative granulation tissue bleed to cause mural hematomas or dissecting hematomas. These can originate immediately following the injurious phase or their development may be delayed weeks, months and even years after the initial onset of SAM [3,5]. Medial sequelae develop from repaired arterial gaps whose lumens may be narrowed by intimal plaques created by the expansion of medial granulation tissue over the adjacent intact intima and by aneurysm walls amply buttressed by granulation tissue. Concurrent thrombi cause further luminal narrowing or luminal occlusions. The granulation tissue that reinforces arterial aneurysms prevents rupture with bleeding but extensive abdominal hemorrhage from sequela aneurysms can occur-an exception to the ischemic axiom of reparative phase SAM. Finally sequelae may generate fibromuscular dysplasia (FMD), the type dependent upon the principal location of the reparative granulation tissue in the artery [6]. The organization of the granulation tissue in zones of medial muscle loss alone or in gaps generates medial dysplasia, if found in the tear separation space it fashions perimedial dysplasia and if localized to the adventitia creates adventitial fibroplasia.

The histological and angiographic findings and clinical symptoms in the current case are a classic representation of reparative phase SAM converted to FMD. An assortment of arterial lesions detected both histologically and radiologically is an important diagnostic feature of both injurious and reparative phase SAM. This variety is created by the varying intensities of the two different arterial injurious lesions, their asynchronous maturation, degree and sites of florid repair and segmental distribution [3]. This case illustrates the varying types of lesions typifying reparative phase SAM. These are medial-adventitial tears repaired by granulation tissue that serves as both sites and sources of dissecting and mural hematomas (Figures 8-10). Prior studies of SAM have shown that these lesions are initiated from hemorrhage in the granulation tissue filling the adventitial-medial separation plane rather than from intimal tears [1,3] and as this 
case illustrates, by the unsatisfactory stent attempt, this bleeding can be traumatically induced. We suspect that the cuff surrounding the celiac axis (Figure 1 ) represents a mural hematoma possibly partially or totally filled with granulation tissue. The second lesion is sequela aneurysms that are well repaired by granulation tissue that forms plaques over adjacent intact arterial intima and may bedeck surviving medial islands (Figure 8). These frequently grossly appear as large fusiform aneurysms (Figure 2) formed by two or more adjacent microscopic aneurysms created by the segmental distribution of SAM's gaps. The latter fashions the saw-toothed appearance frequently found in medial FMD. The third lesion is the occurrence of medial granulation tissue with fibrosis repairing areas of mediolysis Figures 9 and 11). This change always commences in the outer media and may extend inwardly to involve some or all of the inner media. It is often patchy because of the survival of muscle cells in zones of mediolysis. Arterial stenosis, the forth lesion, is cause by the aforementioned lesions and reparative intimal plaques (Figures 3, 5 and 11). The fifth lesion is the formation of thrombi formed in both the aneurysms and pseudo-lumens of dissecting hematomas with thrombo-embolization developing distal to these lesions.

Organ injury is under appreciated initial manifestation of SAM. It may present in the injurious or reparative phases of SAM alone or accompanied by other arterial lesions as in the present case. In addition to renal infarcts [7], another urologic presentation of SAM is the development of perirenal hematomas caused by bleeding retroperitoneal artery aneurysms [8]. Other reported organ injuries include pancreatic hematomas derived from aneurysms of the splenic artery or its large pancreatic branches $[9,10]$ and ischemic bowel lesions induced by arterial stenosis of colonic arteries [11]. It is these renal and bowel lesions that are mainly responsible for the ischemic symptoms announcing reparative phase SAM. The arterial sequelae creating these lesions are generally limited to a single large artery and/or one of its immediate branches serving or immediately entering the involved organ an observation nicely verified in the current case showing SAM restricted to a single hilar artery and its immediate intrarenal lobar branch. The restricted nature of SAM limits organ damage, such as the focal renal infarct in this case, and is not a cause of massive infarcts or multiple angiographically identified arterial lesions in the periphery of the kidneys or liver. Many arterial sequelae are asymptomatic and as this case illustrates may totally disappear [see figure 14 in reference 1]. The paucity of intestinal lesions reported with FMD of the renal arteries is one observation that challenges the hypothesis that SAM is the precursor of FMD. The angiographic disappearance of SAM's sequelae along with SAM's occasional selective localization explains this phenomenon (see reference [1] for other challenges and their defense).

Judging from the fibrosis of the reparative alterations visualized in the outer media we suspect that this lesion was of some duration. Since there was no history of abdominal hemorrhage, the injurious phase of SAM was apparently subclinical and may have dated back ten years to her prolonged treatment with ritodrine. This Beta-2 tocolytic agonist, pharmacologically similar to feneterol may also neuromodulate the peripheral sympathetic nervous system to synthesis and release norepinephrine [12]. The myocardial necrosis reported in infants following treatment with this agent [13] is putatively apoptotic created by the stimulation of the cardiomyocyte B1-adrenergic receptors by norepinephrine released from cardiac ganglia. We suspect that ritodrine also has the capability of stimulating the synthesis and release of norepinephrine from varicosities on the efferent branch of the sympathetic nerves of the splanchnic arteries to initiate SAM. However, given the absence of detectable myocardial damage in the patient, the impossibility of accurately dating the arterial fibrosis, and the absence of angiographic studies predating the patient's onset of symptoms, an alternative possibility is that ritodrine was not the direct agent causing SAM but its prolonged administration sensitized the medial muscle alpha-1 receptors to agonists causing SAM. These agonists may have been unknowingly introduced at a later date perhaps in over-the counter pharmacologic remedies containing alpha-1 adrenergic agents or by meat products poisoned by the repartitioning agents ractopamine or clenbuterol [2].

The renal vein fibrotic lesion, consistent with the repair of an endothelin-1 (ET-1) induced venous angiopathy, suggests ET-1 may have potentiated the pressor activity of norepinephrine $[1,4]$. This lesion frequently accompanies abdominal SAM. ET-1, synthesized in capillaries in the adventitia of the adjacent abdominal arteries, cross-talking with the norepinephrine produced in the ganglia of the efferent sympathetic nerve endings in the outer arterial wall potentially can convert a norepinephrine induced vasoconstrictor physiological stimulus into SAM [1]. However, the role played by ET-1 in SAM is still uncertain. The possibility that it may be additionally released in response to the formed sequelae was the rational for treatment in this patient with the ET-1 antagonist bosentem. The disappearance of all the remaining angiographic sequelae following 5 months of treatment with this agent supports this notion. However, this needs confirmation since spontaneous resolution of sequela aneurysms is reported in SAM [3]. Finally, the pressor effects of norepinephrine may be further intensified because of the increased sensitivity of the alpha-1 adrenergic receptors to adrenaline and by inference to the agonists causing SAM reported in premenopausal females 
[14]. This finding possibly accounts for the female gender preference in FMD.

\section{CONCLUSION}

This case illustrates how SAM, presented in a premenopausal female exposed to Beta-2 tocolytic agonists capable of causing the release of norepinephrine, can be transformed into clinical FMD by transiting a subclinical injurious phase before its reparative sequelae initiate the renal infarction and ischemic bowel symptoms that clinically announced this entity.

\section{ACKNOWLEDGEMENTS}

The principal author wishes to thank and express his appreciation to Dr. Teresa Lozano for her help in gathering and forwarding to me the clinical, radiological and histologic data for this article and to Jeffery Forsyth for his invaluable help with computer problems and Jason Slavin for his assistance in formatting the digital images. No funding agent played a role in the preparation or writing of this manuscript.

\section{COMPETING INTERESTS}

Authors have declared that no competing Interests exist.

\section{AUTHORS CONTRIBUTIONS}

First author wrote the first draft of the manuscript, photographed the pathologic changes and assembled the references, the second author contributed the radiological findings and descriptions, the third, forth and sixth authors managed the clinical aspects of this manuscript and the fifth author supplied the pathologic material for this study. All authors have read and approved the final manuscript.

\section{CONSENT}

The patient has consented to be presented in this manuscript and indeed is one of its co-authors.

\section{REFERENCES}

[1] Slavin, R.E. (2013) Segmental arterial mediolysis: A clinical-pathologic review, its role in fibromuscular dysplasia and description and differential diagnosis of the masquerader-muscular artery cystic necrosis. World Journal of Cardiovascular Disease, 3, 64-81. http://dx.doi.org/10.4236/wjcd.2013.31013

[2] Slavin, R.E. and Yaeger, M.J. (2011) Segmental arterial mediolysis-an iatrogenic vascular disorder induced by ractopamine. Cardiovascular Pathology, 21, 334-338. http://dx.doi.org/10.1016/j.carpath.2011.09.003

[3] Slavin, R.E. (2009) Segmental arterial mediolysis: Course, sequelae, prognosis, and pathologic-radiologic correlation. Cardiovascular Pathology, 18, 352-360. http://dx.doi.org/10.1016/j.carpath.2008.09.001

[4] Slavin, R.E. and Inada, K. (2007) Segmental arterial mediolysis with accompanying venous angiopathy. Report of 3 new cases and comments on the role of endothelin-1 in its pathogenesis. International Journal of Surgical Pathology, 15, 121-134.

http://dx.doi.org/10.1177/1066896906297684

[5] Hashimoto, T., Degachi, J., Endo, H. and Miyata, T. (2008) Successful treatment tailored to each splanchnic arterial lesion due to segmental arterial mediolysis. Journal of Vascular Surgery, 48, 1338-1344. http://dx.doi.org/10.1016/j.jvs.2008.05.056

[6] Slavin, R.E., Saeki, K., Bhagavan, B. and Maas, A.E. (1995) Segmental arterial mediolysis: A precursor to fibromuscular dysplasia? Modern Pathology, 8, 287-294.

[7] Ito, N., Kuwahara, G., Sukehiro, Y. and Teratgani, H. (2012) Segmental arterial mediolysis accompanied by renal infarction and pancreatic enlargement: A case report. Medical Case Reports, 6, 307.

http://dx.doi.org/10.1186/1752-1947-6-307

[8] Mistune, K., Kikuchii, M., Kondo, H., Moriyama, Y., Tsuchiya, T., Mashahiro, N., et al. (2008) Rupture pseudoaneurysm of the renal artery associated with segmental arterial mediolysis. Hinyokika Kiyo, 54, 489-491.

[9] Naitoh, I., Ando, T., Shimohira, M., Nakazawa, T., Hayashi, K., Okumura, F., et al. (2010) Hemosuccus pancreaticus associated with segmental arterial mediolysis successfully treated by transarterial embolization. Journal of the Pancreas (online), 11, 625-629.

[10] Obara, H., Matsubara, K., Inoue, M., Nakatsuka, S., Kuribayashi, S. and Kitagawa, Y. (2011) Successful endovascular treatment of hemosuccus pancreaticus due to splenic artery aneurysm associated with segmental arterial mediolysis. Journal of Vascular Surgery, 54, 14881491.

[11] Wang, J.J.L. and Huang, T.W. (1994) Ischemic colitis caused by isolated dissecting aneurysm of the left colic artery: A presumed case of segmental mediolytic arteriopathy. Journal of the Formosa Medical Association, 93, 715-719.

[12] Burniston, J.G., Tan, L.B. and Goldspink, D.F. (2006) Relative myotoxic and hemodynamic effects of B-agonists fenoterol and clenbuterol measured in conscious unrestrained rats. Experimental Physiology, 91, 1041-1049. http://dx.doi.org/10.1113/expphysiol.2006.035014

[13] Katz, V.L. and Seeds, J.W. (1989) Fetal and neonatal cardiovascular complications from B-sympathomimetic therapy for tocolysis. American Journal of Obstetrics \& Gynecology, 161, 1-4. http://dx.doi.org/10.1016/0002-9378(89)90219-6

[14] Schouwenberg, B.J., Rietjens, S.J., Smits, P. and de Galan, B.E. (2006) Effect of sex on the cardiovascular response to adrenaline in humans. Journal Cardiovascular Pharmacology, 47, 155-157. http://dx.doi.org/10.1097/01.fjc.0000198519.28674.cc 